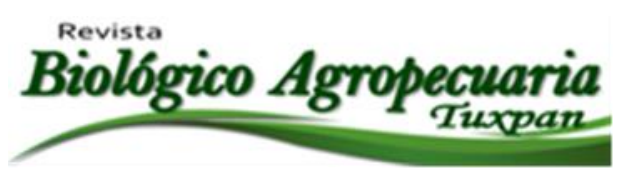

\title{
Control in vitro de Mocis latipes presente en pastizales de Tantoyuca, Veracruz
}

In vitro control of Mocis latipes present in grasslands of Tantoyuca, Veracruz Hernández-Hernández Mayreli ${ }^{1}$; González-Cárdenas Julio César²; Arrieta-González Armando ${ }^{1}$; SilvaMartínez Karla Lissette ${ }^{1 凶}$; Del Ángel-Piña Oscar ${ }^{1}$

${ }^{1}$ Tecnológico Nacional de México-Instituto Tecnológico Superior de Tantoyuca, Vera-cruz, México, C.P. 92100. ${ }^{2}$ Universidad Veracruzana - Facultad de Ciencias Biológico y Agropecuarias, Tuxpan, Veracruz, México, C.P. 92870

${ }^{凶}$ Autor para correspondencia: karla.silva@itsta.edu.mx

Recibido: $15 / 07 / 2020$
Aceptado: 15/10/2020

\section{RESUMEN}

Esta investigación tuvo como objetivo evaluar en laboratorio el potencial biocontrolador de Metarhizium anisopliae sobre Mocis latipes presente en pastos ganaderos del Municipio de Tantoyuca, Veracruz. La investigación se desarrolló en el año 2019; los microorganismos nativos se obtuvieron de 48 muestras compuestas de suelo, tomadas dentro de las unidades de producción ganaderas sin perturbación o poco transitadas, estas muestras se inocularon gusanos trampa (larvas de Galleria mellonella). Se aislaron tres cepas de Metarhizium anisopliae en medio agar-papa-dextrosa. Cada una de estas se inoculó sobre un grupo de 10 insectos adultos de Mocis latipes. Las pruebas de patogenicidad realizadas mostraron que los aislados nativos del género Metarhizium anisopliae, son promisorios para el control de Mocis latipes, con los que se puede plantear un control efectivo de esta especie a pesar de ser una plaga esporádica y voraz. Es necesario aplicar estas cepas en campo y evaluarlas en otras especies de insectos como mosca pinta (Aeneolamia spp., Prosapia spp.), las cuales son una plaga de importancia económica que afecta las gramineas forrajeras cultivadas en la Huasteca Veracruzana. El hongo Metarhizium anisopliae, el cual habita en diferentes tipos de suelo, podría ser usado para el control de plagas por su potencial como agente biocontrolador. Existen pequeñas poblaciones de este hongo en el suelo por lo que es necesario reproducirlas masivamente.

Palabras clave: Metarhizium anisopliae, control biológico, pastos.

\begin{abstract}
This investigation aimed to evaluate in vitro Metarhizium anisopliae as a biological control agent of Mocis latipes, which is present in the grassland of farms in the municipality of Tantoyuca, Veracruz. The research was developed in 2019, the native microorganisms were obtained from 48 soil samples taken in the animal production units, in not disturb areas or not affected places by human activities. The samples were inoculated in larvae of Galleria mellonella. Three Metarhizium anisopliae stock were
\end{abstract}


isolated in potato-dextrose-agar medium. All of them were inoculated in groups of 10 mature Mocis latipes. The pathogenic test done showed that the isolated native stocks of Metarhizium anisopliae are a potential biological control agent of Mocis latipes. This pathogenic fungus could be used to design effective control programs to this pest, dispite $M$. lapites is not present all over the year but it is voracious. It is necessary to evaluate these $M$. anisopliae stocks in situ, with other pests like Aeneolamia spp and Prosapia spp, because of their economic impact and the damage caused to the forage grasses cultivated in the Huasteca Veracruzana. The fungus Metarhizium anisopliae, which is present in different kinds of lands, could be used to control pests because of its potential as a biocontrol agent. There are small populations of this fungus in the soil, therefore it is necessary a massive reproduction.

Keywords: Metarhizium anisopliae, biological control, forage.

\section{INTRODUCCIÓN}

Tantoyuca, Veracruz es uno de los municipios de mayor importancia del sector ganadero de la región, principalmente se basa en el aprovechamiento de los recursos forrajeros de pastos naturales e introducidos con un total de $58 \%$ de uso del suelo destinado a pastizales (INEGI, 2007). El recurso forrajero es la fuente alimenticia más económica con la que se cuenta disponible en nuestro medio; sin embargo, la introducción continuada de nuevas especies de pastos y forrajes como vía fundamental de alimentación de la masa ganadera ha creado en los últimos años una nueva problemática en su producción: el enfrentamiento a un alto número de plagas las cuales limitan considerablemente el rendimiento y desarrollo de algunos de estos nuevos cultivos (Pezo-Quevedo, 2018). Tradicionalmente el control de estas plagas se hace mediante varias aplicaciones de productos químicos, con efectos adversos en el ambiente y a la salud humana, por lo que es importante encontrar una forma de combate efectivo y económico, mediante el uso de especies autóctonas establecidas y adaptadas a los ecosistemas de origen, que existen en pequeños números poblacionales (Gutiérrez, 2016). En este contexto y con el propósito de mostrar una alternativa amigable de la ganadería con el medio ambiente y la sociedad actual y futura, el presente trabajo tuvo como objetivo buscar alternativas no químicas para el control de plagas que conjuntamente con los sistemas de silvopastoreo permitan disminuir el impacto ecológico negativo de la actividad ganadera. Por ello en el presente trabajo el objetivo fue evaluar el potencial biocontrolador de Metarhizium anisopliae sobre larvas de Mocis latipes en laboratorio.

\section{MATERIALES Y MÉTODOS}

El presente trabajo se realizó en el municipio de Tantoyuca y se ubica entre los paralelos $21^{\circ} 06^{\prime}$ y $21^{\circ} 40^{\prime}$ de latitud norte: los meridianos $97^{\circ}$ $59^{\prime}$ y $98^{\circ} 24^{\prime}$ de longitud oeste; altitud entre 10 y 300 m (INEGI, 2010).

Colecta de muestras de suelo

Se colectaron muestras de suelo provenientes de 48 unidades de producción ganaderas con la finalidad de buscar hongos entomopatógenos, estas fueron colectadas en suelos poco perturbados (tierra de monte) y también de 
zonas en donde se tiene antecedentes de ataque de plagas a los pastos, además se tomaron muestras de suelo de montaña cerca de los predios ganaderos. Se tomaron de cinco muestras compuestas a una profundidad de 20 $\mathrm{cm}$ en cada uno con un peso de $250 \mathrm{~g}$ aproximadamente, estas fueron colocadas en una bolsa de plástico.

Procesamiento de la muestra

Las muestras fueron trasladadas al laboratorio de control biológico de la Facultad de Ciencias Biológicas y Agropecuarias perteneciente a la Universidad Veracruzana. En el laboratorio estas fueron tamizadas en malla de $2 \mathrm{~mm}$ de abertura y se colocaron en un recipiente. Se realizaron cuatro repeticiones de cada una de las muestras tomadas.

Uso de gusanos trampas

Para la búsqueda de hongos entomopatógenos a partir del suelo obtenido de los predios cercanos a los potreros, se utilizaron larvas de Galleria mellonella utilizado como gusanos trampas tomadas de una cría artificial en el laboratorio de control biológico de la Facultad de Ciencias Biológicas y Agropecuarias de la Universidad Veracruzana Campus Tuxpan. Se colocaron cinco larvas por cada una de las muestras compuestas de suelo, se observaban cada 24 horas, para asegurar que estuvieran en contacto constante con el suelo así como a humedad requerida además se mantuvieron bajo temperatura contante de $28{ }^{\circ} \mathrm{C}$ (Doberski y Tribe, 1980).

\section{Aislamiento de hongos entomopatógenos}

Transcurrido ocho días de haber colocado los gusanos "trampa" en las muestras de suelo, se comenzó a extraer los gusanos que tuvieran muestra de presencia de desarrollo micelial, colocándose en cámaras húmedas con la finalidad de proporcionarle las condiciones óptimas de temperatura y humedad como lo recomienda Hatting et al., 1999. Se revisaban día a día con la finalidad de observar desarrollo micelial. Las cepas obtenidas se sembraron en un medio de cultivo de papa, dextrosa y agar (PDA), hasta obtener cepas puras.

Identificación de los hongos aislados

De los hongos que se aislaron en el medio de cultivo PDA, estos se identificaron basados en sus estructuras morfológicas, coloración del micelio y velocidad de crecimiento. Se utilizaron claves taxonómicas de Humber (1997; 2012).

Colecta manual del gusano falso medidor (Mocis latipes)

Para la colecta de Mocis latipes se utilizaron pinzas para tomarlos suavemente a modo de no dañarlos y vasos de plástico para ahí capturarlos. Una vez capturados a los recipientes de plástico se les colocó papel estraza en el fondo, los recipientes se etiquetaron y se taparon con una tela llamada "tul" que es semitransparente también conocida como organza, la cual se colocó a nivel de la boca del vaso y se adhirió con ligas o cinta, se colocaron de 5 gusanos vivos por recipiente y en cada recipiente se introdujeron hojas de pasto estrella (Cynodon plectostachyus) para alimentar a los gusanos $\mathrm{y}$ se humedecieron ligeramente y de esta forma asegurar que lleguen vivos. Estas muestras se colocaron en una caja de cartón sin tapar, posteriormente se llevaron al laboratorio de la Universidad Veracruzana, durante el traslado se asperjó agua de botellón que para mantenerlos hidratados, el agua restante fue absorbida por el papel

estraza. 
Evaluación de las cepas de hongos aislados

Con la finalidad de comprobar que los hongos aislado de gusanos tuvieran capacidad infectiva a plagas de los pastos, estos después de aislados se inocularon a larvas de Mocis latipes, para ello se inocularon a una concentración de $1 \mathrm{x}$ $10^{7}$, concentración utilizada como infectiva a insectos. Para la inoculación se utilizaron crecimiento de los hongos en cajas Petri con siete días de maduración, se raspó una caja Petri con el hongo con la ayuda de un porta objeto y se agregó a $100 \mathrm{~mL}$ de agua destilada estéril en donde con la ayuda de una cámara Neubahuer se ajustó a la concentración anteriormente señalada. Se utilizaron cuatro larvas por hongo, se colocaron en el interior del vaso precipitado y se dejó por un minuto con la finalidad de que el hongo permaneciera en contacto con la larva de Mocis latipes.

\section{RESULTADOS}

En este trabajo se aislaron cepas de hongos entomopatógenos asociados a suelos de montañas poco perturbados, denominadas MT1, MT2 y MT3. De acuerdo con Ramírez et al. (2014), los hongos entomopatógenos constituyen el grupo de mayor importancia en el control biológico de insectos plaga, encontrándose presentes en forma natural en el medio ambiente, en el suelo, en restos de cultivos, sobre los cadáveres de insectos, obteniendo su nutrición de otros organismos o de materia orgánica.

Aislamiento de hongos a partir de larvas de Galleria mellonella

A partir de larvas infectadas (gusanos atrampa) que mostraron en su superficie el crecimiento micelial, los aislamientos presentaron de manera inicial un crecimiento muy similar en forma circular iniciando con una fase no mensurable macroscópicamente, seguida de una fase de crecimiento acelerado (hasta 5 a $7 \mathrm{~mm}$ ) pasada esta etapa, presento crecimiento lento (1 a $2 \mathrm{~mm}$ ).

El aspecto de las colonias se presentó de modo algodonoso de superficie plana, coloración de verde oliva a verde oscuro, considerando estas características coinciden con los descritos por Humber (1997; 2012) para Metarhizium anisopliae, a partir de estas muestras se logró aislar y purificar tres cepas (MT1, MT2 y MT3).

Pruebas de efectividad patogénica

Las cepas aisladas de $M$. anisopliae resultaron patogénicas a Mocis latipes, las larvas inoculadas presentaron después de cinco días sobre la superficie el hongo $M$. anisopliae de acuerdo con las características anteriormente mencionadas. Se realizaron reaislamiento de manera directa del micelio del hongo y se sembró en medio de cultivo PDA, a los 10 días de crecimiento del hongo se observaron las esporas en el microscopio corroborando así la presencia del hongo M. anisopliae. El tiempo de manifestación de micosis obtenidos por las cepas nativas, es un factor importante para el control de insectos (Gutiérrez et al., 2004). Estas pruebas demostraron que los aislamientos nativos del género Metarhizium, son promisorios para el control de Mocis latipes, por lo que es necesario realizar pruebas en campo, $M$. latipes es una plaga regular en esta zona pero voraz. Derivado de estos resultados es importante realizar aplicaciones e inundaciones a los pastos ganaderos inmediatamente cuando se presenten las condiciones de clima que requiere $M$. latipes e inmediatamente después del primer individuo encontrado. Las larvas de $M$. latipes estudiados en este trabajo han sido reportados atacando a 
diferentes tipos de pastos de importancia ganadera, entre ellos: Cynodon plectostachyus, Urochloa brizantha, Megathyrsus maximus, especies sobre las cuales esta larva ejerce una gran presión por la actividad de forrajeo en los sistemas productivos de importancia ganadera (Chaput, 2015), lo que pone de manifiesto la importancia de esta plaga en la zona.

\section{DISCUSIÓN}

Metarhizium es un hongo que se encuentra en todo el mundo, siendo muy estudiado por diversos investigadores por su capacidad patogénica contra plagas agrícolas (Medina et al., 2020). Las especies nativas pueden ser altamente patogénicas alcanzando un índice de moralidad del $60 \%$ y esporulación del $80 \%$, como lo demostraron Rodríguez y colaboradores en 2006 al utilizar cepas de Metarhizium anisopliae y Beauveria bassiana sobre Tuta absoluta. Para alcanzar un $90 \%$ de mortalidad de la población de individuos tratados en un periodo de 15 días se requiere incrementar la dosis (Valle et al., 2020). Sin embargo diversos resultados de investigaciones muestran que aislamientos de $M$. anisopliae pueden ser aún más patogénicos superando el $80 \%$, como lo reportaron Torres et al. (2014) al demostrar la utilidad de cultivos monospóricos y su caracterización patogénica para la selección de aislamientos con potencial bioinsecticida sobre la palomilla de la cera $G$. mellonella. Así mismo Obando et al. (2013) sus resultados reportan mortalidades entre el 76,0\% y $90,7 \%$ sobre el estado adulto de Aeneolamia varia (Hemiptera: Cercopidae). Es importante destacar que los aislamientos nativos obtenidos directamente del insecto hospedero son más propensos a ser patogénicos (Srisukchayakul et al., 2005), así como cuando provienen del respectivo cultivo en el que se encuentra la plaga (García et al., 2011). Lo anterior sugiere que la germinación de esporas en aislamientos nativos depende del insecto hospedero (Srisukchayakul et al., 2005).

Diversos autores reportan que a mayor concentración conidial aumenta la tasa de mortalidad (Solarte et al., 2012; Bautista y González, 2005; Loureiro, 2004). Estudio realizado por Lezamaen et al. (2005) en el que demostraron que Metarhizium puede ser combinado con insecticidas químicos como el Triclorfón, aplicado en maíz para combatir gusano cogollero. Otro estudio realizado por Kassab et al. (2014), mostraron compatibilidad entre $M$. anisopliae y Tiametoxam aplicados en condiciones de campo, ambos muy utilizados en Brasil.

\section{CONCLUSIONES}

Las condiciones climáticas del año 2019 favorecieron la presencia de Mocis latipes y la ausencia de Mosca pinta o salivazo en pastizales de la región, esta última plaga de importancia económica. Se recomiendan investigaciones mediante el uso de $M$. anisopliae para el desarrollo de una formulación que pueda ser aplicada en campo ya que este hongo, que se encuentran comúnmente en suelos pero en bajas poblaciones, puede ser capaz de infectar a poblaciones Mocis latipes, por lo que es conveniente continuar con la investigación..

Los resultados presentados en este estudio permiten contar con una colección de nuevas cepas $M$. anisopliae, caracterizadas a nivel macroscópica y patogénicamente causando daño a Mocis latipes bajo condiciones de laboratorio. 


\section{AGRADECIMIENTOS}

Los autores agradecen el financiamiento del Consejo Nacional de Ciencia y Tecnología (CONACYT), así como al Tecnológico Nacional de México y; al Laboratorio de control biológico de la Universidad Veracruzana de la Facultad de Ciencias Biológicas y Agropecuarias por las facilidades brindadas.

\section{LITERATURA CITADA}

Chaput, P. (2015). Manual del Protagonista Pastos y Forrajes. Vol. 35 anivers. Pastos Y Forrajes. Managua: Catholic Relief services.

Doberski, J. W., \& Tribe, H. T. (1980). Isolation of entomogenous fungi from elm bark and soil with reference to ecology of Beauveria bassiana and Metarhizium anisopliae. Transactions of the British Mycological Society, 74(1), 95-100. https://doi.org/10.1016/S0007$\underline{1536(80) 80013-1}$

García, G. C., González, M. M. B., \& Bautista, M. N. (2011). Patogenicidad de aislamientos de hongos entomopatógenos contra Spodoptera frugiperda (Lepidoptera: Noctuidae) y Epilachna varivestis (Coleoptera: Coccinellidae)/Pathogenicity of isolates of entomopathogenic fungi against Spodoptera frugiperda (Lepidoptera: Noctuidae) and Epilachna varivestis (Coleoptera: Coccinellidae). Revista Colombiana de Entomología, 37(2), 217.

Gutiérrez, M. E. M., Cárdenas, Y. G., \& Lequerque, A. (2016). El género Metarhizium Sorokin y su aplicación en el control de insectos plagas/ genus Metarhizium Sorokin and the microbial control of pests insects. Revista Cubana de Ciencias Biológicas, 5(2), 15.

Gutiérrez-Ramírez, A., Robles-Bermudez, A., Santillan-Ortega, C., Ortíz-Catón, M., \& Cambero-Campos, O. J. (2013). Control biológico como herramienta sustentable en el manejo de plagas y su uso en el estado de Nayarit, México. Revista Bio Ciencias, 2(3).

Hatting, L.J; Humber, R.A.,Poprawski, T.J; Miller, R.M. (1999). A survey of fungal pathogens of aphids from South Africa with special refrence to cereal aphids. Biological control 16 (1): 112. https://doi.org/10.1006/bcon.1999.0731

Humber, R.A. (1997). Fungi: preservation of cultures. In: L. Lacery (Ed.). Manual of Techniques in insect pathology. Academic Press. New York USA. https://doi.org/10.1016/B978-012432555$\underline{5 / 50015-4}$

Humber, R.A. (2012). Identification of entomopathogenic fungi. In: Lacey, L.A. (Ed.), Manual of Techniques in Insect Pathology, Second ed. Academic Press. California,U.S. pp. 151-187. https://doi.org/10.1016/B978-0-12$\underline{386899-2.00006-3}$

Instituto Nacional de Estadística, Geografía, e Informática (INEGI). Censo Agropecuario (2007). Disponible en línea: www.inegi.gob.mx.

Instituto Nacional de Estadística, Geografía, e Informática (INEGI). 2010. Perspectiva Estadística. Veracruz de Ignacio de la Llave. Diciembre de 2010. 
http://cuentame.inegi.org.mx/monografias /informacion/ver/default.aspx?tema=me\& $\mathrm{e}=30$.

Lezama, R., Molina, J., López, M., Pescador, A., Galindo, E., Ángel, C. A., \& Michel, A. C. (2005). Efecto del hongo entomopatógeno Metarhizium anisopliae sobre el control del gusano cogollero del maíz en campo. Avances en Investigación Agropecuaria, 9(1).

Medina, E. Q., Oliveira, A. S., Medina, H. R., \& Rangel, D. E. (2020). Serendipity in the wrestle between Trichoderma and Metarhizium. Fungal Biology. https://doi.org/10.1016/j.funbio.2020.01.0 $\underline{02}$

Ortiz-Catón, M., Alatorre-Rosas, R., ValdiviaBernal, R., Ortiz-Catón, A., MedinaTorres, R., \& Alejo-Santiago, G. (2011). Efecto de la temperatura y humedad relativa sobre el desarrollo de los hongos entomopatógenos. Revista bio ciencias, 1(2).

Pezo Quevedo, D.A. (2018). Los pastos mejorados: su rol, usos y contribuciones a los sistemas ganaderos frente al cambio climático. CATIE, Turrialba (Costa Rica).

Ramírez, H.G., Granja, A.Z., Aguila, E.T., \& Cantoral, M.T. (2014). Manual de producción y uso de hongos entomopatógenos. Laboratorio de entomopatógenos SCB-SENASA.

Rodríguez, M., Gerding, M., \& France, A. (2006). Selección de aislamientos de hongos entomopatógenos para el control de huevos de la polilla del tomate, Tuta absoluta Meyrick (Lepidoptera: Gelechiidae). Agricultura técnica, 66(2), 151-158. https://doi.org/10.4067/S0365$\underline{28072006000200005}$

Solarte, J. A. M., Pardey, A. E. B., Valderrama, U. C., Cobo, N. C. M., \& Gil, C. A. M. (2012). Eficacia de Metarhizium anisopliae para controlar Aeneolamia varia (Hemiptera: Cercopidae), en caña de azúcar/Efficacy of Metarhizium anisopliae to control Aeneolamia varia (Hemiptera: Cercopidae), in a sugar cane. Revista Colombiana de Entomología, 38(2), 177.

Torres de la Cruz, M., Cortez Madrigal, H., Ortiz García, C. F., Cappello García, S., \& Pérez de la Cruz, M. (2014). Cepas monospóricas de Metarhizium anisopliae y su patogenicidad sobre Galleria mellonella en Tabasco, México. Revista mexicana de ciencias pecuarias, 5(2), 171-180.

https://doi.org/10.22319/rmcp.v5i2.3223

Valle, S., Caicedo, W., \& Masapanta, L. (2020). Eficacia de un aislamiento nativo de Metarhizium anisopliae (TI6301) para el control de garrapatas (Rhipicephalus microplus) adultas en condiciones in vitro. 
Copyright (c) 2020 Mayreli Hernández Hernández, Julio César González Cárdenas, Armando Arrieta González, Karla Lissette Silva Martínez y Oscar Del Ángel Piña

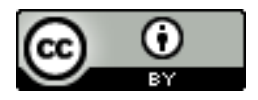

Este texto está protegido por una licencia CreativeCommons $\underline{4.0}$.

\footnotetext{
Usted es libre para Compartir — copiar y redistribuir el material en cualquier medio o formato - y Adaptar el documento —remezclar, transformar y crear a partir del material - para cualquier propósito, , incluso para fines comerciales, siempre que cumpla la condición de:

Atribución: Usted debe dar crédito a la obra original de manera adecuada, proporcionar un enlace a la licencia, e indicar si se han realizado cambios. Puede hacerlo en cualquier forma razonable, pero no de forma tal que sugiera que tiene el apoyo del licenciante o lo recibe por el uso que hace de la obra.

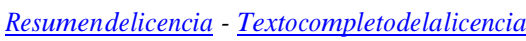

